\title{
Analysis of second wave of COVID-19 cases in Nepal with a logistic model
}

\author{
Radha Krishna Joshi', Sarita Bhatt ${ }^{2}$, Tika Ram Lamichhane ${ }^{3}$, Madhav Prasad Ghimire ${ }^{4}$ \\ ${ }^{1,2}$ Graduate Student, ${ }^{3}$ Assistant Professor, ${ }^{4}$ Associate Professor, Central Department of Physics, Tribhuvan University, \\ Kirtipur-44613, Kathmandu, Nepal
}

Background: COVID-19, caused by SARS-CoV-2, is a newly identified highly infectious disease. It has affected almost every country including Nepal causing a pandemic situation. Most of the properties of SARS-CoV-2 are not known and still under intense investigation. Due to high mutation rate, it reappears in many countries in the form of new variant. In Nepal, second wave impact of COVID-19 is mainly caused by newly found delta variant of SARS-CoV-2. In this case, the mathematical modelling is noted to play important role to understand control strategies for the spread of coronavirus. Aims and Objective: To analyze the second wave impact by modelling the data of COVID-19 cases in Nepal. Materials and Methods: We have analyzed COVID-19 daily cases and deaths reported by Ministry of Health and Population, Government of Nepal from April 1 to May 31, 2021. A logistic model has been used to present the trend line of COVID-19 infection in Nepal, based on the law of population growth developed by Verhulst. Results: The results show a good fit between observed and predicted data by logistic model as indicated by coefficient of determination having value near to unity. The point of inflection from the logistic model predicted a maximum of 9951 daily new cases. The maximum number of cumulative cases estimated at the end of second wave was found to be 307293 with $95 \%$ confidence interval. Conclusion: Logistic model properly describes the growth of COVID-19 cases with time. This type of data modelling and analysis will be very useful in predicting the upcoming trend of COVID-19 in Nepal as a basis for making health policy management by the government.

\section{Access this article online}

Website:

http://nepjol.info/index.php/AJMS

DOI: 10.3126/ajms.v12i10.38763

E-ISSN: 2091-0576

P-ISSN: 2467-9100

Copyright (c) 2021 Asian Journal of Medical Sciences

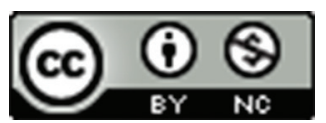

This work is licensed under a Creative Commons Attribution-NonCommercial 4.0 International License.

Key words: COVID-19; SARS-CoV-2; Data modelling; Logistic model; Second wave

\section{INTRODUCTION}

The worldwide pandemic recently reported from Wuhan, China is due to newly identified $\beta$-coronavirus and the disease caused by it is named as coronavirus disease 2019 (COVID-19) named by World Health Organization (WHO). ${ }^{1}$ This disease first noticed in China on 31 December (2019), spreads throughout the globe causing pandemic situation. ${ }^{2}$ Actually, coronaviruses are a group of ribonucleic acid (RNA) viruses which are responsible for respiratory tract infections in humans and some other animals. ${ }^{3,4}$ In human beings, firstly the case of this virus appeared to a person who worked at South China
Seafood market in Wuhan, China. ${ }^{5}$ This virus is genetically related to severe acute respiratory syndrome coronavirus (SARS-CoV) having higher reproduction rate. So, it has got official designation from the International Committee on Taxonomy of Viruses (ICTV) as severe acute respiratory syndrome coronavirus 2 (SARS-CoV-2). ${ }^{6}$

Coronaviruses are a family of RNA viruses that belongs to coronaviridae family. This family is sub-divided into 4 subgroups; alpha, beta, gamma and delta coronavirus. The first two are mainly found in mammals while others are in many birds. ${ }^{7-9}$ SARS-CoV has been detected in bats of China, Europe and Africa around 2002. Similarly, in 2012 
a new type of coronavirus, i.e., middle-east respiratory syndrome coronavirus (MERS-CoV) was reported. It has higher case fatality rate than SARS type coronavirus. ${ }^{10,11}$ Many analysis showed that human coronavirus is most likely related to SARS-CoV that is found in bats. It was believed that SARS-CoV-2 was transmitted in human beings from bats. Though this virus is less pathogenic than SARS-CoV and MERS-CoV, it has high potential of human-to-human transmission which is the most challenging issue of COVID-19. ${ }^{12}$

Coronavirus, one of the largest among RNA viruses, has size ranging from 65-125 nanometer in diameter and 26-32 kilobases in length. ${ }^{1,3,7}$ The major constituents of these viruses are proteins, lipids and single stranded RNA. Coronavirus has crown like spikes made of proteins in its outermost surface which mediates coronavirus entry into the host cell. ${ }^{1,13,14}$ Many studies suggest that the patients of COVID-19 have different symptoms. Most of the individuals suffering from COVID-19 is found to have dry cough, fever, fatigue and breathing problems as reported by WHO. ${ }^{15}$ Besides this, other symptoms included by Center for Disease Control and Prevention are temporary loss of sense of smell, diarrhea, sore throat and headache. ${ }^{16}$ COVID-19 spreads easily when healthy person comes into close contact with infectious person. It may transmit due to small droplets produced by infected person's coughing/ sneezing, aerosol (containing virus) in air and contaminated surfaces. ${ }^{17,18}$

The most common techniques for the diagnosis of COVID-19 are rapid diagnosis test (RDT) and reverse transcriptase polymerase chain reaction (RT-PCR) test. Due to less accuracy of RDT, RT-PCR is used for the confirmative test. ${ }^{19,20}$ Beside this, antigen test can be used at the point of care. It is a biochemical test used to detect the presence of a specific viral antigen.

Some of the protective measures of COVID-19 are to maintain physical distancing, wear mask in public places, avoid contact with others and wash hand frequently. ${ }^{21}$ If we are able to maintain physical distancing, the transmission chain of coronavirus can be broken.

In Nepal, the first confirmed case (a man) of COVID-19 was reported on 23 January, 2020. He was a PhD student at Wuhan University, China. He was kept in quarantine for 8 days and found negative for COVID-19 on January 31. The second case (reported on $23 \mathrm{March}$ ) was a 19-years old girl who had returned from France. Since then, the disease spread gradually to others. ${ }^{22,23}$ Lockdown was applied in the country to control the spread of COVID-19 starting from March 19, 2020 and government declared suspension of all educational classes by physical means, postponement of examinations, halt of government services, transport restriction and prohibiting the gathering of more than 25 people together. ${ }^{24} \mathrm{Nepal}$ shares open border with India and there is no restriction to cross the border without passport. This became one of the major reason for the spread of COVID-19 in Nepal. Likewise, many Nepalese work or go for higher education in different countries. Due to mobility of people COVID-19 was spread in Nepal during first wave. ${ }^{25}$ The first confirmed case of Nepal is also a first case of South Asia. Most of South Asian countries mainly focused on individuals arriving from the affected countries. Unfortunately, controlling them was not enough to control spread of COVID-19. ${ }^{26}$ At the end of first wave, there were 277461 confirmed cases out of which 272851 were recovered and 3031 were died of COVID-19 in Nepal. After declining COVID-19 infection in Nepal for a few months, it started rising suddenly in the beginning of second wave. Since March 2021, an unexpected exponential growth in daily COVID-19 cases in India was reported. Many researches show that this is due to the spread of highly infectious double mutant variant of SARS-CoV-2 found in India named as delta-variant. ${ }^{27}$ Second wave of COVID-19 started in Nepal due to open border problem. Sampling analysis showed that about $97 \%$ of the new cases were due to delta-variant. Due to highly transmittable and more lethal nature of this delta-variant, the health condition of the affected people became very critical as compared to the first wave of COVID-19 so that the infection rate and mortality rate raised significantly.

As scientists are working on a number of vaccines and drugs, many of them are in clinical trial. Some vaccines were approved recently by WHO. The vaccination mobilizes our immune system against SARS-COV-2. The probability of being infected from COVID-19 was reported to reduce for the individuals who was injected two doses of the vaccine. ${ }^{28}$ At present, all the countries have begun their vaccination program using a type of vaccine developed from the biotechnological approaches. ${ }^{29}$ Due to emergence of various new variants (B.1.1.7, ${ }^{30}$ B.1.617.2 ${ }^{27}$ ) of SARS$\mathrm{CoV}-2$, vaccine efficacy is reduced. For this reason, the global acceptability of vaccine is limited. Even then vaccination increases the immunity to some extent thereby reducing the transmission risk and mortality rate. Since there is no particular treatment yet, the best way to study rise and fall of COVID-19 cases is data modelling. The data modelling is widely used to predict the possible impact on the people from the trend line of COVID-19 infection.

\section{MATERIALS AND METHODS}

Due to adverse effect of COVID-19 in global health, it becomes a matter of interest in the field of research. 
At present there are various modelling for the study of different disease with facilitating computational tools/ software. ${ }^{31}$ Currently, compartmental models are widely used for the study of infectious disease. But large number of assumptions reduces the scope of such models. ${ }^{32}$ In the beginning, the increasing levels of COVID-19 suggests that it has exponential tendency. ${ }^{33}$ Exponential function can only account for the growing nature. So, here we present an applied mathematical model, i.e. logistic model for the study of COVID-19 cases in Nepal, which is based on the law of population growth developed by Verhulst. ${ }^{34}$ According to Verhlust, the rate to change of population parameter $N(t)$ is given by the differential equation

$$
\frac{d N(t)}{d t}=c N\left(1-\frac{N}{k}\right)
$$

where $c$ and $k$ are constants. This gives the solution as a logistic equation.

$$
N(t)=\frac{k}{1+\mathrm{e}^{-c\left(t-t_{0}\right)}}
$$

Here, $N(t)$ is cumulative number of cases, $c$ is the growth rate, $k$ is the final size and $t_{0}$ is time at point of inflection after which logistic curve begins to be flat slowly. The fitting ability of model can be determined by coefficient of determination, which is given by

$$
R^{2}=1-\frac{\sum_{j}\left(N_{j}-\widehat{N}_{j}\right)^{2}}{\sum_{j}\left(N_{j}-\bar{N}\right)^{2}}
$$

where $N_{j}$ represents the actual cumulative confirmed cases, $\widehat{N}$, is the predicted cumulative confirmed cases and $\bar{N}$ is average cumulative confirmed cases. Its value lies between 0 and 1 . Greater the value of $R^{2}$ better will be the model.

The observed data for COVID-19 in Nepal were collected from the site of Ministry of Health and Population, Government of Nepal. ${ }^{35}$ The complete data set of the daily cases was collected from April 1 to May 31, 2021 in order to study the second wave impact of COVID-19 in Nepal. The values of four parameters $\left(k, c, t_{0}\right.$ and $\left.R^{2}\right)$ defined in Equation 1 and 2 were calculated by using logistic regression tool of Desmos Graphing Calculator 2021.

\section{RESULTS}

As of 31 May 2021, there have been 561302 confirmed cases of COVID-19 with 106470 active cases, 447446 recoveries and 7368 deaths out of 3057424 RT- PCR tests in Nepal. In the data collection period for second wave of COVID-19, the comparison of cumulative number of confirmed cases, recoveries, deaths and active cases is as shown in Figure 1. It suggests that the curves for both confirmed and recovered cases are in rapid growth indicating a large number of daily new confirmed cases as well as new recoveries. The curve for the death cases is found to be in lazy growth while comparing with other curves. This is because of the number of daily new deaths, which is increasing but in small manner. The curve for active cases is changing with time, i.e. at first it is increasing but after some time it remains same (neither increasing nor decreasing) and finally it begins to decrease. This changing behavior of the curve for active cases is the direct consequence of extension of lockdown and changing number of tests per day.

Second wave of COVID-19 in Nepal started from the first week of April, 2021. The daily infection rate was intensely increasing. One of the major reason for this rapid growth of new cases was previously removed lockdown and prohibition which were applied due to emergence of COVID-19 in Nepal. Second lockdown was implemented in many regions of the country which were at higher risk starting from the last week of April. Due to announcement of lockdown many people started to return home. There were lack of respect for social distancing. The disregard of the strong advice against the public gatherings caused health risks. As a result, confirmed cases of COVID-19 infection were increased rapidly as reflected by the curve with increasing slope (Figure 1).

The fitted curve of the logistic model (following equation 2) for cumulative confirmed cases of second wave in Nepal is as shown in Figure 2. The observed COVID-19 cases are represented by dotted line while the predicted cases using logistic model is represented by solid line. It indicates that growth rate of the total cases in Nepal resembles with that of logistic curve.

Before April 2021, there were few confirmed cases $(<100)$ per day. Towards the beginning of April, the number of cases rapidly increases reaching about 500 new cases per

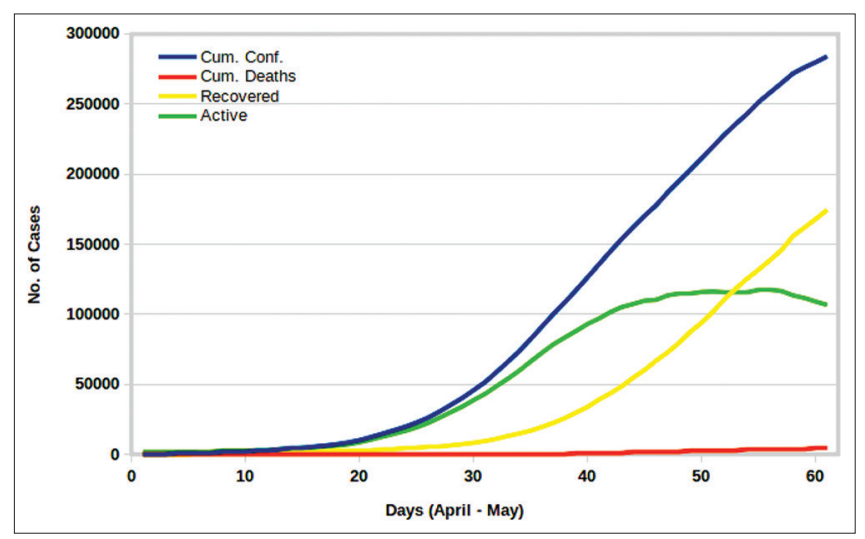

Figure 1: Status of COVID-19 in Nepal from April 1 to May 31, 2021 
day. As the growth rate was large, the number of daily cases reached around 5000 towards the end of April, 2021. Due to lack of control in mobility of people the situation get worst, indicated by 9000 new cases per day until mid-May. The major reasons are the negligence of the people and not implementation of lockdown in the beginning of second wave unlike to first wave. Further, the new delta $(\delta)$ variant of SARS-COV-2 is found to be more strainful than other variants. This causes the impact of second wave much higher than expected. These features are clearly observed as seen in the Figure 3. The logistic model shows a good fit $\left(R^{2}=0.99\right)$ with the observed confirmed cases of COVID-19 in Nepal. In this model, as the saturation begins, the growth rate slows down and curve deviates from exponential growth. At this stage, the model is approximately linear in nature. Once the saturation get completes, growth of the model stops forming horizontal asymptote and the corresponding value of population gives rise to final size for COVID-19, which is known as carrying capacity of the logistic model. This capacity depends on the variation of number of cases with time.

The coefficient of determination represents an efficient relative association between observed cases and predicted

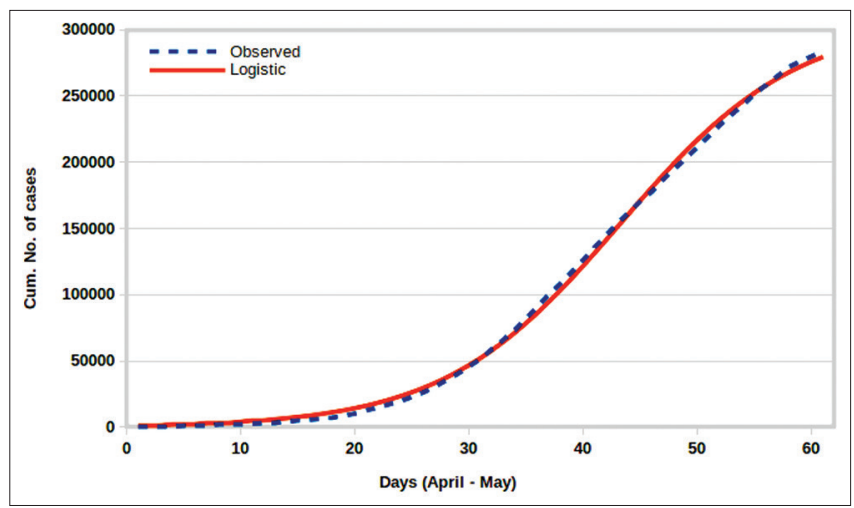

Figure 2: Fitting of COVID-19 cases in Nepal using logistic model

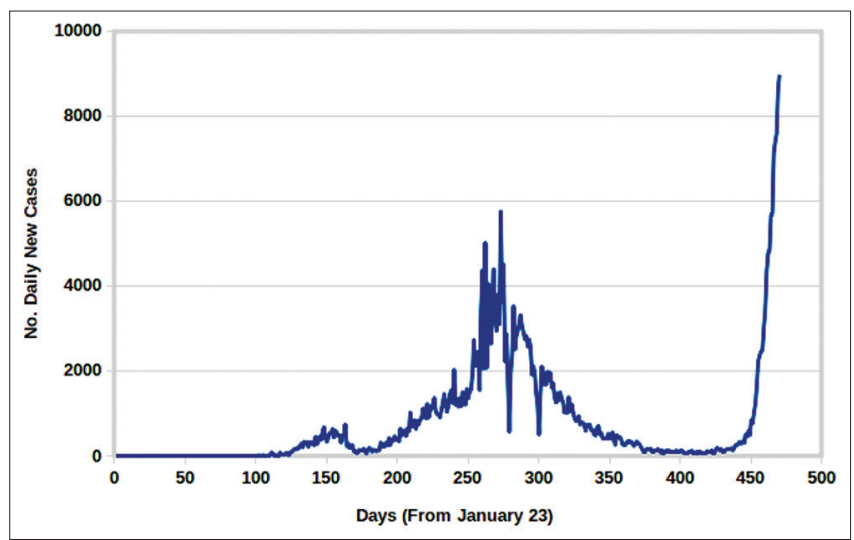

Figure 3: Comparison of first and second wave COVID-19 cases in Nepal cases by logistic model. The calculated parameters from logistic growth model are used to forecast cases of COVID-19 in Nepal based on the present trend of increasing number of cases. The maximum number of cases estimated at the end of second wave was found to be 307293 with $95 \%$ confidence interval. The estimated cases of COVID-19 for Nepal are much less than the observed cases in highly affected countries like USA, Russia, India, Italy, France, Germany and Spain. For a developing country like Nepal, it is not easy to handle the estimated cases and to control the spread of disease. If the growth rate increases, the results may not be true and final size could be greater than the predicted one.

Figure 4 shows the estimated value of cumulative confirmed cases of COVID- 19 in Nepal by logistic model. The curve formed is sigmoid in nature as a characteristic feature of logistic model. Also, the maximum number of daily new cases estimated by the application of logistic model is 9951 by May 14 (44 ${ }^{\text {th }}$ day) which is the point of inflection for logistic model. After this, daily new cases begins to decrease, i.e. saturation begins and hence the curve starts to flatten indicating the decreasing infection rate of COVID-19. In the Figure 4, the upper and lower limits of $95 \%$ confidence interval are represented by green and yellow colors respectively.

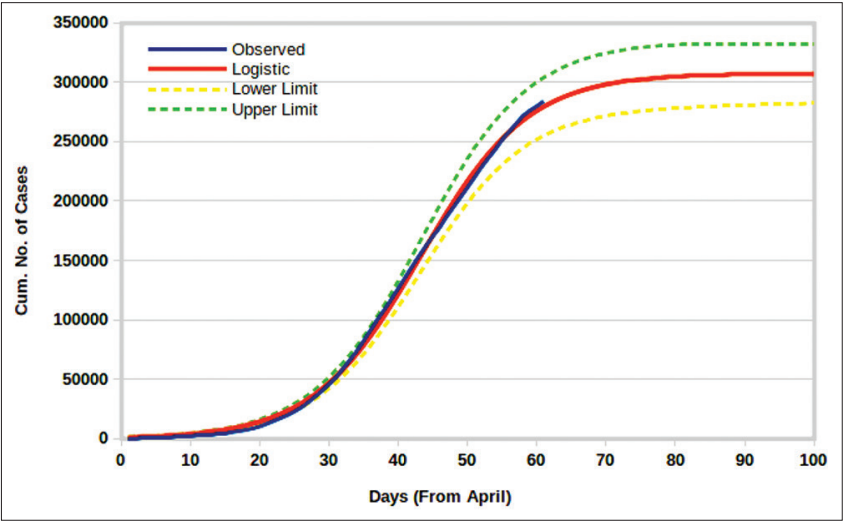

Figure 4: Application of logistic model to predict COVID-19 cases in Nepal

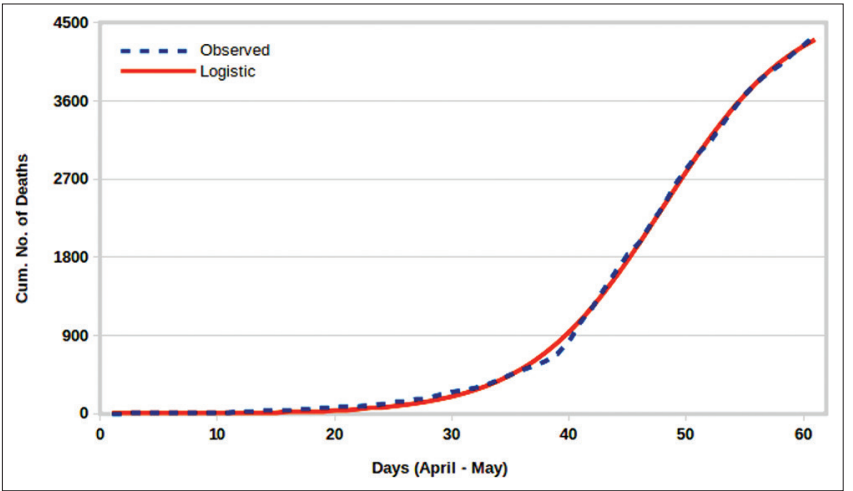

Figure 5: Fitting of COVID-19 death cases in Nepal using logistic model 
In Nepal, the first death due to COVID-19 was reported on May 16, 2020 nearly four months (114 days) after the appearance of the first case. With the statistics of second wave, the number of daily death cases are only few but was increasing day by day. As of May 31 (2021), the estimated case fatality ratio was $1.53 \%$ which is larger than that for the first wave $(1.09 \%)$ estimated at the end of March, 2021. This ratio is used to assess the health care capacity of a country or region in response to infectious disease. Not only for cumulative confirmed cases, but also for cumulative number of deaths, logistic model can be used. The fitted curve of logistic model (Equation 2) for cumulative deaths is as shown in Figure 5. The dotted line represents the observed deaths whereas solid line indicates predicted deaths by logistic model. The observed deaths and the predicted deaths by logistic model are in strong agreement with each other. This model is used to forecast number of deaths due to COVID-19 in Nepal on the basis of present infection rate. The final estimated size for death cases was found to be 4750 with $95 \%$ confidence interval limit. It estimates a maximum of 208 deaths per day. The calculated value of coefficient of determination $R^{2}=0.99$ suggests that there was a good fit between observed deaths and predicted deaths by logistic model.

\section{DISCUSSION}

Modelling and forecasting of COVID-19 pandemic are very challenging task as it is caused due to newly found SARS-CoV-2 and its natural history, modes of transmission and mutation are unknown. Our model is based on existing data and it is designed to fit development of curve for the cases. Dash (2020), studied spread of COVID-19 in Wuhan city and Hubei province, China using logistic model for the period of January 29 to March 20, 2020. They fitted data for confirmed cases, deaths and recovered cases and found that the model fits data with $R^{2}$ in between 0.85 to 0.95 for all regions. This suggests that logistic model is an appropriate model for the study of COVID-19. In our case, results are more accurate as indicated by greater value of $R^{2}$ than their study. ${ }^{36}$ Jie et al., analyzed COVID-19 infection in China using three different mathematical models namely logistic model, Bertalanffy model and Gompertz model. Out of these three models, they found that the fitting effect of logistic model is the best one. The value of $R^{2}$ they obtained is nearly same with our result. ${ }^{37} \mathrm{Nepal}$ (2020) used Gompertz and logistic model for modelling of the first wave of COVID-19 in Nepal by analyzing data in the period January to October, 2020. He found that the logistic model is more appropriate than Gompertz model for the modelling of COVID- 19 data in Nepal. ${ }^{38}$ The value of $R^{2}$ and growth rate parameter calculated by his model were less than the finding of our work. Torrealba-Rodriguez et al., used mathematical models (logistic, Gompertz) and computational model (Artificial Neural Network) for the study of COVID-19 in Mexico. These three models fitted data well with $R^{2}>0.99$ and is used to forecast the cases. In case of logistic model, their growth rate parameter is nearly same as our model, but point of inflection is too late than ours. ${ }^{39}$ Similarly, Attanayake et al., used phenomenological models (logistic, exponential, Gompertz and Weibull) for the modelling of COVID-19 epidemics in Sri Lanka, Italy, USA and Hubei province of China. For these countries, the logistic model resulted to strong fitting between observed and estimated cases with $R^{2}$ greater than $0.98 .{ }^{40}$

\section{CONCLUSION}

On the basis of available data, we used a logistic model to estimate the COVID-19 cases and deaths in Nepal. The result of modelling shows a good compatibility between estimated and observed cases with $R^{2}=0.99$ for both confirmed cases and deaths. Thus, logistic model properly describes the growth in the number of COVID-19 cases with time. As the second wave impact of COVID-19 in Nepal, the estimated final size of cumulative confirmed cases and deaths are 307293 and 4750, respectively with 95\% confidence interval. The point of inflection from the logistic model predicts a maximum of 9951 daily new cases. If the infection rate remains same, then situation will be under control. However, if the infection rate is increased, Nepal's health care system must be improved to deal with upcoming critical cases of COVID-19. As the vaccination reduces spread of COVID-19, it is necessary to distribute the approved vaccines in all parts of the country equally. Further, to control the spread of COVID-19, the transmission chain of coronavirus must be broken by social distancing or any other means. Our predicted results from the model are under the precondition that the situation (such as prevention and control measures, testing order and social distancing strategies) remains same.

\section{REFERENCES}

1. Shereen MA, Khan S, Kazmi A, Bashir N and Siddque R. Covid-19 infection: Origin, transmission, and characteristics of human coronaviruses. J Adv Res. 2020; 24:91-98.

https://doi.org/10.1016/j.jare.2020.03.005

2. Hua $\mathrm{J}$ and Shaw R. Corona virus (Covid-19) infodemic and emerging issues through a data lens: The case of China. Int $\mathrm{J}$ Environ Res Public Health. 2020; 17:2309. https://doi.org/10.3390/ijerph17072309

3. Lu R, Zhao X, Li J, Niu P, Yang B, Wu H, et al. Genomic characterization and epidemiology of 2019 novel coronavirus: implications for virus origins and receptor binding. Int J Environ Res Public Health. 2020; 395:565-574.

https://doi.org/10.1016/S0140-6736(20)30251-8 
4. Morens DM, Breman JG, Calisher CH, Doherty PC, Hahn PC, Hahn BH, Keusch GT, et al. The origin of covid-19 and why it matters. Am J Trop Med Hyg. 2020; 103:955-959.

https://doi.org/10.4269/ajtmh.20-0849

5. Zhang L, Shen F, Chen F and Lin Z. Origin and evolution of the 2019 novel coronavirus. Clin Infect Dis. 2020; 71:882-883. https://doi.org/10.1093/cid/ciaa112

6. Guo YR, Cao QD, Hong ZS, Tan YY, Chen SD, Jin HJ, et al. The origin, transmission and clinical therapies on coronavirus disease 2019 (covid-19) outbreak an update on the status. Mil Med Res. 2020; 7:11.

https://doi.org/10.1186/s40779-020-00240-0

7. Perlman S and Netland J. Coronaviruses post-SARS: update on replication and pathogenesis, Nat Rev Microbiol. 2009; 7:439-450. https://doi.org/10.1038/nrmicro2147

8. Hu D, Zhu C, Ai L, He T, Wang Y, Ye F, et al. Genomic characterization and infectivity of a novel SARS like coronavirus in Chinese bats. Emerg Microbes Infect. 2018; 7:154. https://doi.org/10.1038/s41426-018-0155-5

9. Cui J, Han H, Streicker D, Li G, Tang X, Shi Z, et al. Evolutionary relationships between bat coronaviruses and their hosts. Emerg Infect Dis. 2007; 13:1526-1532. https://doi.org/10.3201/eid1310.070448

10. Tu C, Crameri G, Kong X, Chen J, Sun Y, Yu M, et al. Antibodies to SARS coronavirus in civets. Emerg Infect Dis. 2004; 10:22442248.

https://doi.org/10.3201/eid1012.040520

11. Wernery U, Lau SKP and Woo PCY. Middle East respiratory syndrome (MERS) coronavirus and dromedaries. Vet J. 2017; 220:75-79.

https://doi.org/10.1016/j.tvjl.2016.12.020

12. Chan JFW, Yuan S, Kok KH, Wang KK, Chu H, Yang J, et al. A familial cluster of pneumonia associated with the 2019 novel coronavirus indicating person-to-person transmission: a study of a family cluster. Lancet. 2020; 395:514-523.

https://doi.org/10.1016/S0140-6736(20)30154-9

13. Li F. Structure, function, and evolution of coronavirus spike proteins. Annu Rev Virol. 2016; 3:237-261.

https://doi.org/10.1146/annurev-virology-110615-042301

14. Lamichhane TR and Ghimire MP. Research on COVID-19 from biophysical perspective. Tribhuvan University Journal. 2020; 34:1-14.

https://doi.org/10.3126/tuj.v34i0.31535

15. Wang Z, Yang B, Li Q, Wen $L$ and Zhang R. Clinical features of 69 cases with coronavirus disease 2019 in Wuhan, China. Clin Infect Dis. 2020, 71:769-777.

https://doi.org/10.1093/cid/ciaa272

16. Kimball A, Hatfield KM, Arons M, James A, Taylor J, Spicer K et al. Asymptomatic and presymptomatic SARS-CoV-2 infections in residents of a long term care skilled nursing facility-King County, Washington, March 2020. MMWR. 2020; 69:377-381. https://doi.org/10.15585/mmwr.mm6913e1

17. Bao L, Gao H, Deng W, Lv Q, Yu H, Liu M, et al. Transmission of severe acute respiratory syndrome coronavirus 2 via close contact and respiratory droplets among human angiotensin- converting enzyme 2 mice. J Infect Dis. 2020; 222:551-555. https://doi.org/10.1093/infdis/jiaa281

18. Ge ZY, Yang L, Xia JJ, Fu XH and Zhang YZ. Possible aerosol transmission of covid-19 and special precautions in dentistry. J Zhejiang Univ-Sci B. 2020; 21:361-368.

https://doi.org/10.1631/jzus.B2010010
19. Andrey DO. Head-to-head accuracy comparison of three commercial covid-19 igm/igg serology rapid tests. J Clin Med. 2020; 9:2369.

https://doi.org/10.3390/jcm9082369

20. Tahamtan A and Ardebili A. Real-time RT-PCR in COVID-19 detection: issues affecting the results. Expert Rev Mol Diagn. 2020; 20:453-454.

https://doi.org/10.1080/14737159.2020.1757437

21. Hamner L, Dubbel P, Capron I, Ross A, Jordan A, Lee J, et al. High SARS-CoV-2 attack rate following exposure at a choir practice Skagit County, Washington, March 2020. MMWR. 2020; 69:606-610.

https://doi.org/10.15585/mmwr.mm6919e6

22. Piryani R M, Piryani S and Shah JN. Nepal's response to contain COVID-19 infection. JNHRC. 2020; 18:128-134. https://doi.org/10.33314/jnhrc.v18i1.2608

23. Poudel K, Subedi P. Impact of COVID-19 pandemic on socioeconomic and mental health aspects in Nepal. Int J Soc Psychiatry 2020; 66:748-755. https://doi.org/10.1177/0020764020942247

24. Mahato P, Tamang P, Shahi P, van Teijlingen E, Aryal N, Regmi P, et al. Impact of COVID-19 pandemic on socioeconomic and mental health aspects in Nepal. EJMS 2020; 2:105-110. https://doi.org/10.46405/ejms

25. Chalise HN. COVID-19 situation in Nepal. Arch Psychiatr Ment Health 2020; 4:34-34. https://doi.org/10.29328/journal.apmh.1001015

26. Bhutta ZA, Basnyat $B$, Saha $S$ and Laxminarayanan R. COVID-19 risks and response in South Asia, BMJ. 2020; 369: m1190. https://doi.org/10.1136/bmj.m1190

27. Kuppalli K, Gala P, Cherabuddi K, Kalantri SP, Mohanan M, Mukherjee B, et al. India's COVID-19 crisis: a call for international action. The Lancet. 2021; 397: 2132-2135. https://doi.org/10.1016/S0140-6736(21)01121-1

28. Benenson S, Oster $\mathrm{Y}$, Cohen MJ and Nir-Paz R. BNT162b2 mRNA Covid-19 vaccine effectiveness among health care workers. N Engl J Med. 2021; 384:1775-1777. https://doi.org/10.1056/NEJMc2101951

29. Alvarado-Socarras JL, Vesga-Varela AL, Quintero-Lesmes DC, Fama-Pereira MM, Serrano-Diaz NC, Vasco M, et al. Perception of COVID-19 vaccination amongst physicians in Colombia, January 2021. Vaccines. 2021; 9(3):287. https://doi.org/10.3390/vaccines9030287

30. Sah R, Khatiwada AP, Shrestha S, Bhuvan KC, Tiwari R, Mohapatra RK, et al. COVID-19 vaccination campaign in Nepal, emerging UK variant and futuristic vaccination strategies to combat the ongoing pandemic. Travel Med Infect Dis. 2021; 41:102037.

https://doi.org/10.1016/j.tmaid.2021.102037

31. Douglas JV, Bianco S, Edlund S, Engelhardt T, Filter M, Gunter T, et al. STEM: an open source tool for disease modeling. Health Secur. 2019; 4:291-306. https://doi.org/10.1089/hs.2019.0018

32. Liu M, Cao J, Liang J and Chen MJ. Epidemic dynamics modeling and analysis. Epidemic-logistics Modeling: A New Perspective on Operations Research, Springer, Singapore. 2019; 13-44. https://doi.org/10.1007/978-981-13-9353-2_2

33. Dudala SR, Sau A, Appina B, Srivastava MAS and Mohapatra A. A preliminary prediction of COVID-19 cases in India by April 2020 using exponential mathematical modelling. Natl J Res 
Community Med. 2020; 9:1-5.

https://doi.org/10.26727/NJRCM.2020.9.1.001-005

34. Verhulst PF. Notice sur la loi que la population poursuit dans son accroissement. Corresp Math Phys. 1838; 10:113-121.

35. COVID-19 pandemic data/Nepal medical cases, Wikipedia (accessed on June 1, 2021). URL: https://en.wikipedia.org/wiki/ Template: COVID-19_pandemic_data/Nepal_medical_cases

36. Dash M. Analyzing the propagation of the coronavirus epidemic: The case of Wuhan in Hubei province, China. Epidem Int. 2020; 5:39-43.

https://doi.org/10.24321/2455.7048.202008

37. Jia L, Li K, JiangY, GuoX and Zhao T. Prediction and analysis of coronavirus disease 2019. arXiv preprint arXiv:2003.05447 (2020).
38. Nepal SR. An analysis of COVID-19 cases in Nepal: a modelling approach. JIST. 2020; 25:80.

https://doi.org/10.3126/jist.v25i2.33744

39. Torrealba-Rodriguez $\mathrm{O}$, Conde-Gutierrez RA and HernandezJavier AL. Modeling and prediction of COVID-19 in Mexico applying mathematical and computational models. Chaos Solitons Fractals. 2020; 138:109946.

https://doi.org/10.1016/j.chaos.2020.109946

40. Attanayake A, Perera SSN and Jayasinghe S. Phenomenological modelling of COVID-19 epidemics in Sri Lanka, Italy, the United States, and Hebei province of China. Comput Math Methods Med. 2020; ID 6397063.

https://doi.org/10.1155/2020/6397063

Authors Contribution:

RKJ- Statistical analysis and interpretation, prepared first draft of manuscript, revision of the final manuscript; SB- Statistical analysis, literature review and preparation of first draft of manuscript, revision of the final manuscript; TRL- Concept and design of the study, statistical analysis and interpretation, preparation of manuscript; MPG- Concept and design of the study, coordination, interpretation, revision of the manuscript

Work attributed to:

Central Department of Physics, Tribhuvan University, Kirtipur, Kathmandu, Nepal.

Orcid ID:

Mr. Radha Krishna Joshi - (iD https://orcid.org/0000-0002-2273-8212

Ms. Sarita Bhatt - (1) https://orcid.org/0000-0003-3151-1607

Dr. Tika Ram Lamichhane - (1) https://orcid.org/0000-0002-3422-0808

Dr. Madhav Prasad Ghimire - (D) https://orcid.org/0000-0003-2783-4008

Source of Support: Nil, Conflict of Interest: None declared. 